\title{
Extracellular High-Mobility Group Box 1 Protein (HMGB 1) as a Mediator of Persistent Pain
}

\author{
Nilesh M Agalave and Camilla I Svensson \\ Department of Physiology and Pharmacology, Karolinska Institutet, Stockholm, Sweden
}

\begin{abstract}
Although originally described as a highly conserved nuclear protein, high-mobility group box 1 protein (HMGB1) has emerged as a danger-associated molecular pattern molecule protein (DAMP) and is a mediator of innate and specific immune responses. $\mathrm{HMGB1}$ is passively or actively released in response to infection, injury and cellular stress, providing chemotactic and cytokine-like functions in the extracellular environment, where it interacts with receptors such as receptor for advanced glycation end products (RAGE) and several Toll-like receptors (TLRs). Although HMGB1 was first revealed as a key mediator of sepsis, it also contributes to a number of other conditions and disease processes. Chronic pain arises as a direct consequence of injury, inflammation or diseases affecting the somatosensory system and can be devastating for the affected patients. Emerging data indicate that $\mathrm{HMGB1}$ is also involved in the pathology of persistent pain. Here, we give an overview of $\mathrm{HMGB1}$ as a proinflammatory mediator, focusing particularly on the role of HMGBI in the induction and maintenance of hypersensitivity in experimental models of pain and discuss the therapeutic potential of targeting HMGBI in conditions of chronic pain.
\end{abstract}

Online address: http://www.molmed.org

doi: $10.2119 / \mathrm{molmed} .2014 .00176$

\section{INTRODUCTION}

High-mobility group box 1 protein (HMGB1) is a DNA-binding protein that is abundant in the cell nucleus of most mammalian cells. By binding transiently to chromatin, it exerts structural and transcriptional activities (1). HMGB1 can be passively released by injured or necrotic cells or actively secreted; in this way, it serves as a danger-associated molecular pattern molecule protein (DAMP) (2). Besides its essential roles in the nucleus, HMGB1 gains increasing attention as a secreted protein capable of inducing cytokine release, regulating the activity of leucocytes, lymphocytes, epithelial cells, glial cells and neurons. HMGB1 mediates its action via several receptors, including Toll-like receptor (TLR)-2, TLR4 and the receptor for advanced glycation end products (RAGE) (3-5) and additional receptors. HMGB1 has not only been identified as a late mediator of infectious inflammation such as sepsis (6), but has also been implicated as a putative signal involved in the pathogenesis of a variety of noninfectious conditions, including arthritis, colitis, cancer, ischemia, epilepsy and chronic pain $(3,7)$. Here, we describe recent insight into the role of HMGB1 in the induction and maintenance of hypersensitivity in experimental models of pain and discuss the therapeutic potential of targeting HMGB in conditions of chronic pain.

\section{ACUTE AND PERSISTENT PAIN}

Acute pain results from the activation of pain pathways by peripheral stimuli

Address correspondence to Camilla I Svensson, Karolinska Institutet, Von Eulers väg 8, 17177 Stockholm, Sweden. Phone: +46-8-524-87948; Fax: +46-8-310622. E-mail: camilla.svensson@ki.se.

Submitted September 4, 2014; Accepted for publication September 9, 2014; Epub (www.molmed.org) ahead of print September 10, 2014.

\section{The Feinstein Institute for Medical Research

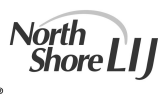
Empowering Imagination. Pioneering Discovery.

of intensities that threaten or lead to tissue damage. As such, the transmission of painful stimuli is an important warning system and activator of learning and memory formation; it helps to prevent injury by evoking a reflex withdrawal from the stimuli and induces complex behavioral strategies to avoid further or repeated contact with such stimuli. Primary sensory neurons that are responsible for the detection and transduction of painful stimuli (for example, cold, heat, mechanical and chemical) are called nociceptors. Persistent nociceptive signaling after tissue damage results in activity-dependent plasticity or a progressive increase in the response of the system to subsequent stimulation, such that mildly noxious (painful) stimuli are perceived as more painful, and nonpainful stimuli may now elicit pain $(8,9)$. This pain hypersensitivity assists in healing of the injured body part by creating a situation that discourages physical contact and movement. Activation of the immune system by tissue injury or infection is a common driver of pain hypersensitivity and is therefore often called "inflammatory pain." Persistent or reoccurring pain, even when it may still serve as 
a protective function, can have a detrimental impact on quality of life and needs to be reduced (for example, in patients with ongoing inflammation or in cases of severe or extensive injury). Furthermore, under some circumstances, pain outlasts its physiological role, and this pain is out of proportion to the initial injury or occurs without any apparent cause. Neuropathic pain is an example of such maladaptive pain, which neither protects the organism nor supports tissue repair $(10,11)$. Neuropathic pain can be induced by direct trauma to a peripheral nerve, disease states such as diabetes mellitus and viral infections and treatments including antiretroviral drugs and chemotherapy agents.

Facilitation of neuronal activation in the peripheral nervous system (peripheral sensitization) and the central nervous system (central sensitization) are common features of persistent pain. Both peripheral and central sensitization are induced and maintained by transcriptional, translational and posttranslational regulation (12). In peripheral sensitization, both a reduction in the threshold for activation and an increased responsiveness of the peripheral ends of the nociceptors can be seen. It is driven by a number of inflammatory mediators, for example, prostaglandins, bradykinin, ATP (adenosine 5 -triphosphate), protons, nerve growth factor and cytokines, released from non-neuronal cells such as fibroblasts, mast cells, neutrophils and macrophages (13). After nerve damage, these mediators can also be released from Schwann cells and damaged axons. The development of central sensitization in persistent pain is characterized by enhancement of excitatory and decreased inhibitory synaptic transmission in the dorsal horn of the spinal cord in a way in which normal inputs begin to produce exaggerated and/or abnormal responses $(14,15)$. These processes involve the phosphorylation of a number of different receptors, including $N$-methyl D-aspartate (NMDA), $\alpha$-amino-3-hydroxy-5-methyl4-isoxazolepropionic acid receptor (AMPA) and/or kainate receptors, which increases synaptic activity, for example, by altering channel opening time and promoting trafficking of receptors to the synaptic membrane (16). Emerging preclinical data implicate three types of glial cells in the development and maintenance of chronic pain: satellite glial cells in the dorsal root and trigeminal ganglia (17-19) and microglia and astrocytes in the central nervous system $(13,20,21)$. Many of the proinflammatory factors that drive peripheral sensitization can be released from local glia cells and neurons in the central nervous system and contribute to central sensitization (22).

\section{HMGB 1}

HMGB1 (previously also called HMG1, amphoterin and p30) is a nonhistone DNA binding protein that is abundant in the cell nucleus. Nuclear HMGB1 acts as a DNA chaperone with DNA binding and bending activities and regulates a number of key DNA events such as DNA replication, repair, recombination, transcription and genomic stability (1). HMGB1 is a 25-kDa protein containing two positively charged DNA-binding domains (HMG box A and B) and a negatively charged C-terminus. The amino sequence of HMGB1 is $99 \%$ identical in mammals. Although predominantly located in the nucleus in most cells at baseline, biologically active HMGB1 can be transported to the cytosol, plasma membrane and extracellular space. HMGB1 release occurs either passively or actively during pathogen invasion and tissue injury. These two major pathways of release differ in terms of inducing event, molecular mechanisms, release kinetics and downstream signaling responses. The passive release of HMGB1 by dead, dying or injured cells is rapid and associated with increased cell permeability $(23,24)$, whereas active secretion initiated by membrane receptor interaction with extracellular products and intracellular signal transduction occurs more slowly $(6,25)$. Active secretion of HMGB1 can be induced in monocytes, macrophages, natural killer cells, dendritic cells, endothelial cells, neurons, astrocytes and mi- croglia (26) after exposure to pathogenassociated molecular patterns (PAMPs) such as lipopolysaccharide (LPS) (6) or stimuli such as interleukin (IL)-1, tumor necrosis factor (TNF) (6), interferons (27-29) and neuropeptide $Y(30)$.

Dissecting the mechanisms of HMGB1 translocation and its compartmentspecific roles is critical to advance the understanding of HMGB1 in physiology and pathophysiology. Whereas the DNA binding domains contain two nuclear localization signals and two nonclassical nuclear export signals (31), HMGB1 lacks a secretory signal peptide and therefore cannot be actively secreted through classical endoplasmic reticulum-Golgi pathways (6). In activated monocytes and macrophages, HMGB1 is acetylated and accumulates in cytoplasmic vesicles, and exocytosis of HMGB1 via nonclassical vesicle-mediated secretory pathways has been demonstrated (25). HMGB1 release is also regulated by other posttranslational modifications including methylation (32) and phosphorylation by calcium/calmodulin-dependent protein kinase and protein kinase C (PKC) (33-35). Recent studies show that during pyroptosis, Janus kinase/signal transducer and activator of transcription (JAK/STAT1)-mediated acetylation is critical in the initial nucleus to cytosol shuttling, and the subsequent extracellular release is controlled by NLRP3 inflammasome and dsRNA-activated protein kinase R (PKR) $(28,36)$. Still, although it is clear that immune cells actively release HMGB1 into the extracellular space and the details surrounding this is starting to emerge, the mechanism of how other cells, including sensory neurons and spinal glial cells, regulate HMGB1 release in response to different signals remains largely unknown.

Once outside the cells, HMGB1 has a broad repertoire of immunological activities that include the induction of $\mathrm{cy}$ tokine production, chemotaxis, angiogenesis, cell proliferation and cell differentiation (2). In addition to effects on immune cells, HMGB1 can modulate the activities of hematopoietic, epithelial 
and neuronal cells (26). With regards to neuronal activity, it has been shown that HMGB1 increases the excitability of primary afferent neurons $(37,38)$, potentiates NMDA-induced hippocampal neuronal activity $(39,40)$ and amplifies neuron-astrocyte reciprocal signaling (37,40-42). HMGB1 mediates its activities by serving as a ligand for several different receptors, including TLR2, TLR4, TLR9, RAGE, CD24-Siglec-10 pathway (Siglec-G in mice) and macrophage antigen-1 (complement receptor consisting of Cd11b and CD18) (4,42-45).

HMGB1 signaling through RAGE mediates chemotaxis, proliferation and differentiation of immune cells and other cells as well as upregulation of cell-surface receptors, including TLR4 and RAGE (2). In contrast, HMGB1-mediated TLR4 signaling is a strong inducer of cytokine production, in a fashion strictly dependent on the redox state of HMGB1 (see below). In addition to direct receptor interactions, HMGB1 can form complexes with selected ligands (for example, LPS, IL-1, bacterial DNA, nucleosomes, CXCL12 and viral RNA) and enhance the responses dramatically when compared with induction by the ligand alone (46-48). The exact mechanism underlying this synergy is not known, although dependence on partner ligand receptor and the independence of TLR2, TLR4 or RAGE has been demonstrated (48).

Recent work has revealed that the extracellular activities of HMGB1 depend on the redox state of three cysteines at positions 23, 45 and 106 within the protein $(49,50)$. When these three cysteines are all reduced (HMGB1 ${ }^{\mathrm{C} 23 \mathrm{hC} 45 \mathrm{hC} 106 \mathrm{~h}}$, allthiol HMGB1), HMGB1 acts as a RAGE ligand (51) and potentiates chemotactic activity via CXCR4 by forming a heterocomplex with the CXCL12 chemokine (49). As the cytosol is generally a reducing environment, HMGB1 is thought to be in the all-thiol state intracellularly and most likely also initially upon release. The extracellular milieu, however, tends to be more oxidizing than the nuclear and cytoplasmic compartment, allowing the formation of disulfide bridges. When
C23 and C45 are engaged in a disulfide bridge and the cysteine in position C106 is in the reduced thiol form (HMGB1 ${ }^{\mathrm{C} 23-\mathrm{C} 45 \mathrm{C} 106 \mathrm{~h}}$, disulfide HMGB1), HMGB1 is stabilized in a conformation in which it functions as a cytokine-inducing TLR4 ligand (50). When HMGB1 is terminally oxidized to contain sulfonyl groups on all cysteines (HMGB1 ${ }^{\mathrm{C} 23 \mathrm{soC} 45 \mathrm{soC} 106 s o}$, sulfonyl HMGB1), it is thought to be nonactive, since this form has not yet been associated with an in vivo function. Notably, it has been shown in models of hepatic inflammation and muscle injury that released HMGB1 changes its redox state from a reduced to first a partially and then a fully oxidized form $(49,50)$. This result implies that the changes in the cellular redox environment regulate the temporal function of HMGB1 so that the different HMGB1 redox forms interact with different receptors, at different phases of the pathophysiological process. Thus, when dissecting the actions of HMGB1, it is critical to consider the redox form of HMGB1. An example of the importance of this is the controversy around the cytokine-inducing activity of HMGB1, potentially introduced by differences in protein preparation and the redox state of the cysteine residues. Commercially available recombinant HMGB1 preparations can contain the reducing agent dithiothreitol, which disrupts the crucial disulfide bond and renders HMGB1 in the all-thiol form (50). These preparations are unable to stimulate cytokine production, and their use in experiments has led to conflicting reports regarding the capacity of HMGB1 to induce inflammation.

\section{BEHAVIORAL EVIDENCE OF NOCICEPTIVE PROPERTIES OF HMGB 1}

Immune and glial cells express RAGE and TLRs and are located in the close vicinity of nociceptors. It is well established that these cells, both in the peripheral and central nervous system, can be activated and release factors that in turn can directly drive or facilitate pain signaling (22,52). However, as TLR4 (53-55) and RAGE $(38,56)$ are also expressed in peptidergic and glutamatergic dorsal root ganglia (DRG) sensory neurons, the machinery allowing HMGB1 to act as a direct modulator of neuronal activity is in place. Mounting evidence indicates that TLRs and RAGE and their associated signaling components contribute to nociceptive signaling, and the blockade of TLRs and RAGE has been shown to reduce hypersensitivity in experimental models of pain $(38,57)$. Furthermore, since HMGB1 forms complexes with other factors and enhances the effect of its partner molecules (for example, IL-1 and LPS) (46-48), one unexplored possibility is that HMGB1 facilitates neuronal activity (directly or indirectly) also by potentiating the actions of such receptors. Thus, there are several direct and indirect ways in which HMGB1 can have an impact on pain signaling.

\section{Peripheral Local Injection/Application of HMGB 1}

The first study indicating that HMGB1 has pronociceptive properties was performed more than 10 years ago. Chacur et al. (58) applied HMGB1 to the sciatic nerve via a pre-implanted indwelling peri-sciatic catheter and found that this induced a dose-dependent reduction in mechanical thresholds against von Frey filament stimulation in rats. Later, a similar observation was made after application of HMGB1 to the sciatic nerve following a blunt dissection to expose the nerve (56). Importantly, HMGB1 also induces pain-like behavior without prior surgery. Subcutaneous injection of HMGB1 to the plantar side of the paw (59) and intraarticular injection of disulfide-HMGB1 to the ankle joint of mice drives mechanical hypersensitivity (Figure 1). There is still limited information on whether HMGB1 induces cold hypersensitivity, but HMGB1 increases sensitivity to heat after intraplantar injection of HMGB1 to the paw (59) and after perisciatic injection, together with a midthigh incision (56), but not when injected via a peri-sciatic catheter (58). Of note, unilateral peri-sciatic injection of high-dose HMGB1 induced bilateral mechanical 


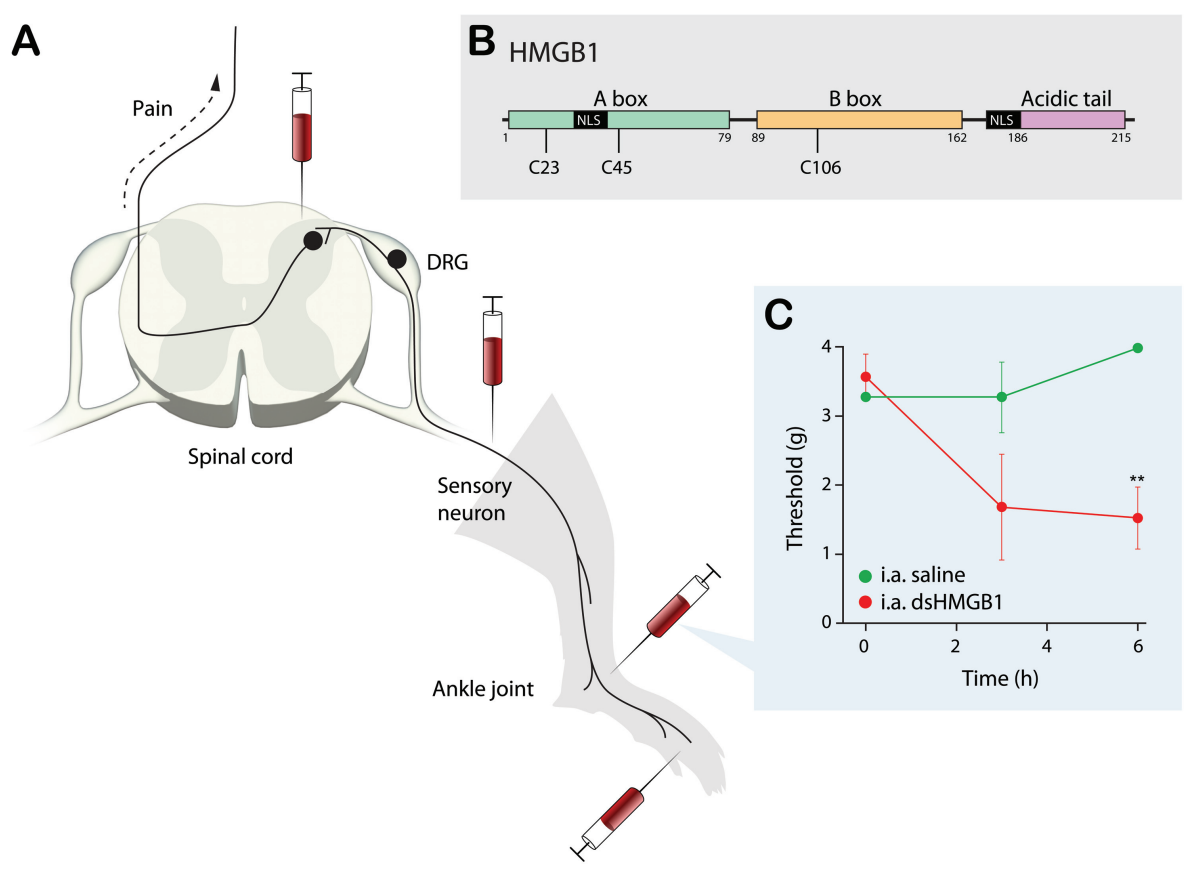

Figure 1. HMGB1 induces pain-like behavior in rodents. (A) Injection of recombinant HMGB1 to the paw, ankle joint, sciatic nerve or intrathecal space evokes pain-like behavior in rodents. (B) The structure of HMGB1 with the two DNA binding domains (the A and B boxes), the acidic C-terminal tail and nuclear location signals (NLSs) outlined. The three cysteine residues that are important for functional activity of HMGB1 are highlighted. (C) Graph shows that intraarticular (i.a.) injection of $1 \mu \mathrm{g}$ disulfide HMGB1, but not saline, induces mechanical hypersensitivity in male Balb/c mice $6 \mathrm{~h}$ after injection. Mechanical sensitivity is assessed by von Frey filaments, and data are presented as threshold (g) with $50 \%$ probability of response. Data represents mean \pm standard error of the mean. $n=5 /$ group. ${ }^{* *} p<0.001$.

hypersensitivity (58), a phenomenon called "mirror pain" (60). Intraperitoneal injection of HMGB1 does not evoke painlike behavior (61), which suggests that HMGB1-induced mirror pain is not caused by systemic redistribution but rather arises from altered processing of incoming sensory information in the central nervous system.

Extracellular HMGB1 is found in joints from animals with adjuvant arthritis and in synovial tissue and fluid from patients with rheumatoid arthritis (62). HMGB1 can induce joint inflammation on its own, as evidenced by the influx of inflammatory cells, thickening of the synovial lining and cytokine production after intraarticular injection of HMGB1 (63). Many of the cytokines that are released after joint inflammation drive nociceptive signaling (22). Thus, pain-like behavior induced by intraarticular injection of HMGB1, as well as injection of HMGB1 elsewhere, could be mediated by direct actions on neurons, or indirectly via the activation of immune cells.

While in vivo studies provide important information about the pronociceptive properties of HMGB1, in vitro studies are crucial in determining whether HMGB1 acts directly on nociceptors. Intriguing studies in primary DRG neuronal cultures using radiometric calcium imaging and patch-clamp electrophysiology showed that HMGB1 drives calcium mobilization and increases the excitability of primary afferent neurons (37). The majority of cells in which HMGB1 induced calcium mobilization also responded to capsaicin; such a response is indicative of the fact that the cells are nociceptive neurons. Furthermore, the ability of disulfide
HMGB1 and all-thiol HMGB1 to activate sensory neurons in DRG cultures has been investigated. Allette et al. (38) found that all-thiol HMGB1 and disulfide HGMB1 evoke calcium transients in $14 \%$ and $8 \%$ of primary DRG neurons, respectively. In addition, patch-clamp recordings showed that all-thiol HMGB1 induced a significant increase in neuronal excitability, which was reduced in the presence of a RAGE antibody (11E6), but not by small molecule antagonists to TLR2 or TLR4 (38). Thus, current data suggest that both disulfide and all-thiol HMGB1 have a direct effect on neuronal excitability. Although experiments examining whether disulfide HMGB1mediated actions on neurons are mediated via TLR4 still need to be performed, all-thiol HMGB1 responses in primary neurons appear to be mediated by RAGE.

\section{Intrathecal Injection of HMGB 1}

The first evidence of a role of HMGB1 in spinal nociceptive signaling was established in 2003 when HMGB1 was injected intrathecally (into the spinal fluid) in rats, and it was revealed that this caused a pronounced lowering of the response threshold to calibrated touch/ pressure stimuli in male rats (64). Subsequent studies demonstrated comparable effects after intrathecal injection of HGMB1 to female and male mice (65). In the later study, it was also shown that the spinal pronociceptive property of HMGB1 depends on its redox state. In the naive animal, spinal injection of HMGB1 induces mechanical hypersensitivity only when it is in the disulfide TLR4-activating, but not the all-thiol or sulfonyl non-TLR4 binding, forms (HMGB1). Moreover, intrathecal injection of disulfide HMGB1, but not all-thiol HMGB1, induced increased levels of mRNA of glial fibrillary acidic protein (Gfap), Cd11b, Tnf, Il1 $\beta$ and monocyte chemoattractant protein $(M c p) 1$, typically found during microglia and astrocyte activation. The nociceptive, cytokine and glial cell activating effect of disulfide HMGB1 was abolished in TLR4-, but not TLR2- or RAGE-deficient, mice, indicat- 
Table 1. Inhibition of HMGB1 in experimental models of pain.

\begin{tabular}{|c|c|c|c|c|c|}
\hline Pain model & Nociceptive assessment & HMGB1 inhibition & $\begin{array}{l}\text { Route of drug } \\
\text { administration }\end{array}$ & Species & $\begin{array}{l}\text { Refer- } \\
\text { ence }\end{array}$ \\
\hline \multicolumn{6}{|l|}{ Nerve injury pain } \\
\hline SNL & Mechanical (VF); thermal (hotplate) & HMGBI Ab & Peri-sciatic & Rat & 56 \\
\hline Nucleus pulposus & Mechanical (VF) & HMGB1 Ab & Intraperitoneal & Rat & 76 \\
\hline $\mathrm{TNI}$ & Mechanical (VF) & Glycyrrhizin & Intraperitoneal & Rat & 37 \\
\hline Diabetes & Mechanical (VF) & HMGB1 Ab & Intrathecal & Mouse & 80 \\
\hline PSNL & Mechanical (VF) & HMGB1 Ab & Intravenous & Rat & 75 \\
\hline \multicolumn{6}{|l|}{ Inflammatory pain } \\
\hline \multirow[t]{2}{*}{ LPS paw } & \multirow{2}{*}{$\begin{array}{l}\text { Mechanical (VF); thermal (thermal } \\
\text { analgesia meter) }\end{array}$} & HMGB1 Ab & Intraplantar & Rat & 59 \\
\hline & & HMGB1 Ab & Intraplantar & Rat & 59 \\
\hline Arthritis & Mechanical (VF) & HMGB1 Ab; Abox & Intrathecal & Mouse & 65 \\
\hline \multicolumn{6}{|l|}{ Other pain } \\
\hline Bone cancer & Mechanical (VF) & HMGB1 Ab & Intrathecal & Rat & 79 \\
\hline $\begin{array}{l}\text { Cyclophosphamide-induced } \\
\text { bladder pain }\end{array}$ & Mechanical (VF) & Thrombomodulin; HMGBI Ab & Intraperitoneal & Mouse & 61 \\
\hline
\end{tabular}

SNL, spinal nerve ligation; VF, von Frey filaments; Ab, antibody; TNI, tibial nerve injury; PSNL, partial sciatic nerve ligation; CCl, chronic constriction induced.

ing that disulfide HMGB1 mediates processes associated with spinal sensitization via TLR4. Intracellular, HMGB1 predominantly exists in the reduced, allthiol state (66), which implies that, to gain spinal nociceptive properties, allthiol HMGB1 has to be converted to disulfide HMGB1.

\section{ROLE OF ENDOGENOUS HMGBI IN NOCICEPTIVE SIGNALING}

It has not been possible to assess the role of HMGB1 in nociception by genetic depletion, because HMGB1 knockout mice die within $24 \mathrm{~h}$ of birth because of severe hypoglycemia, attributed to impaired glucose metabolism (67). In contrast, HMGB1-deficient cells grow normally, and specific HMGB1 depletion in pancreatic or myeloid cells generate viable mice, indicating that HMGB1 is not critical for the baseline function of these cells $(68,69)$. Animals with cell lineage-specific depletion of HMGB1 have not yet been used for pain studies. Instead, agents that inhibit the activity of HMGB1, such as neutralizing anti-HMGB1 antibodies $(70,72)$; the truncated HMGB1A box domain, which antagonizes HMGB1 activities in a yet-unresolved mode $(71,72)$; glycyrrhizin, a natural antiinflammatory and antiviral triterpene in clinical use (73); and thrombomodulin, an endothelial anticoagulant known to sequester HMGB1 and promote its degradation (74), have been used in different experimental models of pain.

\section{Blocking the Action of Peripheral HMGB 1}

Neuropathic pain is a frequent problem after different types of trauma to a nerve. Because HMGB1 is released from injured cells, the hypothesis that HMGB1 is a key player in neuropathic pain is intriguing, and an increasing number of reports support such a relationship. The induction of mechanical hypersensitivity after spinal nerve ligation (ligation of the L4 and L5 spinal nerves) (56), tibial nerve injury (ligation of the tibial nerve) $(37,38)$, partial sciatic nerve ligation (onethird of the sciatic nerve ligated) (75) and application of disc nucleus pulposus on to nerve roots (model of disc herniation) (76) are attenuated by systemic or local administration of HMGB1-neutralizing antibody $(56,75,76)$ or glycyrrhizin $(37)$ (Table 1). Thus, regardless of the exact location and nature of the nerve injury, blocking the actions of peripheral HMGB1 reduces pain-like behavior in experimental models of neuropathic pain, suggesting that HMGB1 has an important role in pain transmission after nerve injury.
The antihyperalgesic effect of HMGB1neutralizing antibodies, which presumably exert their activity extracellularly, supports the notion that HMGB1 is released in response to nerve injury. Although release of HMGB1 after nerve injury has not yet been demonstrated in vivo, increased levels of Hmgb1 mRNA in DRG after spinal nerve ligation (56) and HMGB1 protein in whole-cell DRG extracts after tibial nerve injury (37) have been reported. In addition, immunohistochemical studies showed that HMGB1 levels are upregulated in neurons $(37,56)$ and satellite cells (56) in the DRG after nerve ligation-induced injury and are present in macrophages infiltrating the DRG after application of nucleus pulposus to the nerve (76) (Table 2). The elevated levels of HMGB1 may be an indication that HMGB1 is released from neurons, satellite and/or immune cells after nerve injury and exerts its actions in an autocrine/paracrine effect on neurons or immune cells.

While there are several reports describing a role for peripheral HMGB1 in nerve injury-induced hypersensitivity, only a few studies have focused on the involvement of peripheral HMGB1 in pain conditions of other origins. However, based on two recent studies, in which skin and visceral (bladder) hyper- 
HMGBI AS A MEDIATOR OF PAIN

Table 2. Expression of HMGB1 in DRG and spinal cord.

\begin{tabular}{|c|c|c|c|c|c|c|}
\hline Pain model & Species & Tissue & Method & HMGB1 expression & Antibody & $\begin{array}{l}\text { Refer- } \\
\text { ence }\end{array}$ \\
\hline \multicolumn{7}{|l|}{ Nerve injury pain } \\
\hline SNL & Rat & $\begin{array}{l}\text { DRG } \\
\text { Spinal nerve }\end{array}$ & IHC & $\begin{array}{l}\text { Naive: IR in satellite cells, neurons, Schwann cells } \\
\text { SNL: } \uparrow \text { in most neurons } \\
\text { Naive and SNL: IR cells }\end{array}$ & Rabbit, SHINO-test & 56 \\
\hline Nucleus pulposus & Rat & DRG & WB & Total homogenate: NC & Rabbit, Shino-Test & 76 \\
\hline $\mathrm{TNI}$ & Rat & DRG & $\begin{array}{l}\text { IHC } \\
\text { WB }\end{array}$ & $\begin{array}{l}\text { Naive: nuclear IR in satellite cells, neurons } \\
\text { TNl: } \uparrow \text { in cytoplasm (in neurons) } \\
\text { Total homogenate: NC, extranuclear fraction: } \uparrow\end{array}$ & Rabbit, Sigma-Aldrich & 37 \\
\hline Diabetes & Mouse & Spinal cord & WB & Total homogenate: $\uparrow$ & Rabbit, BD Pharmingen & 80 \\
\hline PSNL & Rat & Spinal cord & $\begin{array}{l}\text { IHC } \\
\text { WB }\end{array}$ & $\begin{array}{l}\text { Naive: IR in microglia, astrocytes, neurons } \\
\text { PSNL: } \uparrow \text { in neurons } \\
\text { Total homogenate: } \uparrow\end{array}$ & Mouse private & 75 \\
\hline $\mathrm{CCl}$ & Rat & Spinal cord & WB & Total homogenate (dorsal horn): $\uparrow$ & Rabbit, BD Pharmingen & 99 \\
\hline $\mathrm{CCl}$ & Rat & Spinal cord & WB & Total homogenate: $\uparrow$ & Rabbit, Abcam & 100 \\
\hline \multicolumn{7}{|l|}{ Inflammatory pain } \\
\hline LPS paw & Rat & DRG & WB & Total homogenate: NC & Rabbit, SHINO-test & 61 \\
\hline Arthritis & Mouse & Spinal cord & $\begin{array}{l}\text { WB } \\
\text { WB } \\
\text { IHC }\end{array}$ & $\begin{array}{l}\text { Total homogenate: NC } \\
\text { Extranuclear fraction: } \uparrow \\
\text { Naive: IR in microglia, astrocytes, neurons }\end{array}$ & Rabbit, Abcam & 65 \\
\hline
\end{tabular}

Other pain conditions

Bone cancer

Rat Spinal cord

WB Total homogenate (dorsal horn): $\uparrow$

Rabbit, BD Pharmingen

79

SNL, spinal nerve ligation; IHC, immunohistochemistry; IR, HMGB1 immunoreactivity; $\uparrow$, increase; WB, Western blot; NC, no change; TNI, tibial nerve injury; PSNL, partial sciatic nerve ligation; $\mathrm{CCl}$, chronic constriction induced.

sensitivity was induced by intraplantar injection of LPS to the paw and intraperitoneal injection of cyclophosphamide, respectively, there are reasons to consider HMGB1 as a contributor to nociception also in conditions of more inflammatory character. Intraplantar injection of LPS to the paw evokes both mechanical and thermal hypersensitivity in rats. This hypersensitivity was reduced in rats pretreated with systemic thrombomodulin. Furthermore, coinjection of LPS with a neutralizing HMGB1 antibody prevented the onset of LPS-induced hypersensitivity (59), which suggests that either LPS drives the release of HMGB1 or HMGB1 forms a complex with LPS and enhances the nociceptive potency of LPS in the same way as the ability of HMGB1 to potentiate the immunostimulatory property of LPS in cultured cells $(77,78)$. Tanaka et al. (61) noted that intraperitoneal injec- tion of cyclophosphamide led to increased levels of Hmgb1 mRNA in DRG and that systemic injection of either thrombomodulin or neutralizing HMGB1 antibodies attenuate cyclophosphamideinduced bladder pain-like behavior, noted as a reduced licking and biting of the skin of the lower abdomen and hypersensitivity in the area of the anus and the urethral opening of mice.

\section{Blocking the Action of Spinal HMGB 1}

The first site of synaptic integration in the pain pathway is located in the dorsal horn of the spinal cord. Changes in the excitability of spinal neurons or in the strength of their synaptic coupling provide the cellular basis for many forms of pathological pain. There are accumulating evidence for spinal glial cells contributing to neuronal excitability in experimental models of pain. Notably, in addition to the ability of HMGB1 to directly or indirectly increase the responsiveness of peripheral primary nociceptors, three recent studies $(65,79,80)$ suggest that HMGB1 also contribute to exaggerated nociceptive signaling by actions at the spinal level.

Bone cancer pain is the most common source of pain in patients with bone sarcomas and metastasis that have spread to the bone. In animals, inoculation of carcinoma cells in the tibia is associated with increased protein expression of HMGB1 in the spinal cord, spinal glial cell activation and cytokine production and painlike behavior. Injection of anti-HMGB1 antibody into the cerebrospinal spinal fluid (intrathecal injection) reversed bone cancer-induced hypersensitivity in a dose-dependent fashion. In addition, blocking the action of HMGB1 reduced cancer-induced IL-1 $\beta$ protein expression 
in the spinal cord (79). The $\mathrm{db} / \mathrm{db}$ mice are used as a model of type 2 diabetes, and these mice develop mechanical hypersensitivity, thought to mimic the painful peripheral neuropathy that is a common complication of type 2 diabetes. Intrathecal injection of neutralizing HMGB1 antibodies reversed not only the mechanical hypersensitivity in the $\mathrm{db} / \mathrm{db}$ mice, but also upregulation of mRNA of inflammatory mediators such as Tnf, Il1 $\beta$, Il6 and Mcp1. On the basis of GFAP protein expression, glial cell reactivity was also reduced in the $\mathrm{db} / \mathrm{db}$ mice after spinal HMGB1 blockage (80). Lastly, in a mouse model of rheumatoid arthritis induced by the systemic injection of collagen type 2 antibodies, it was found that arthritis-induced mechanical hypersensitivity was attenuated by either intrathecal injection of neutralizing HMGB1 antibody or the A box (65). Of note, the collagen antibody-induced arthritis (CAIA) model has two phases of pain-like behavior: one that occurs with the development of joint inflammation and one that persists for weeks after resolution of the initial flare of inflammation. The later phase is thought to be mediated at least in part via mechanisms that are similar to those maintaining nerve injury-induced hypersensitivity $(81,82)$. Blocking the spinal action of HMGB1 reversed both phases of CAIA-induced mechanical hypersensitivity (65). The fact that the prevention of HGMB1-mediated actions in the spinal cord had a beneficial effect in animal models representing very different diseases suggests a wide-ranging involvement of spinal HMGB1 in pathological pain.

In parallel with the notion that effects of neutralizing HMGB1 antibodies after systemic injection point to the presence of HMGB1 in the extracellular space in the periphery, a reduction of pain-like behavior after intrathecal injection of HMGB1 antibodies reveals the extracellular actions of HMGB1 in the spinal cord and suggests that peripheral pathologies may drive spinal HMGB1 release. Indeed, elevated levels of HMGB1 in the spinal cord have been detected in several experimen- tal models of pain. Measuring HMGB1 in whole-cell protein extracts showed elevated levels of HMGB1 protein in the spinal cord after the induction of nerve ligation (75), diabetic neuropathy (80) and bone cancer (79) (Table 2). Primary sensory neurons have their central terminals in the spinal cord; thus, it is possible that HMGB1 is released spinally by injured and/or activated noninjured primary sensory nociceptors. In support of this notion, it has been shown that F11 cells, a DRG neuron/neuroblastoma hybrid cell line, release HMGB1 upon stimulation with potassium chloride (37), indicating that neuronal HMGB1 release may occur in an activity-dependent manner. HMGB1 was detected in the cell culture media and cytosol of BV2 cells, a microglia cell line, in response to LPS stimulation (83-85) and in human astrocytes in response to IL-1 $\beta$ (86). As the activation of spinal glial cells has been implicated in many experimental models of both inflammation and nerve injury-induced pain, microglia and astrocytes are other potential sources for extracellular HMGB1. Furthermore, extracellular HMGB1, independent of the cellular source, may contribute to the activation of non-neuronal cells in the spinal cord and the release of other factors that potentiate spinal nociceptive signal transmission, thereby indirectly contributing to pain hypersensitivity. Both microglia and astrocytes express TLRs and RAGE, and HMGB1 stimulation of primary microglia cell cultures induces increased mRNA levels of Tnf, Il1 $\beta$ and cyclooxygenase (Cox) (87) and the release of IL-6 (88). Furthermore, cultured astrocytes exposed to HMGB-1 produce inflammatory molecules such as COX-2, chemokines and matrix metallopeptidase 9 (MMP-9) $(89,90)$, each promoting nociceptive hypersensitivity. In addition, in gliosomes, glial organelle preparation is suggested to represent a viable system for studies of transmitter release in adult astrocytes; HMGB1 induces glutamate release in a glutamate transporter-dependent fashion (91). Although the exact details of the cellular source of spinal HMGB1 and the cell(s) responsible for driving nociceptive actions of spinal HMGB1 remain to be elucidated, these studies mentioned above consistently point in the same direction, indicating HMGB1 as a proinflammatory factor that regulates spinal pain processing.

\section{INTERPRETING CHANGES OF HMGB 1 EXPRESSION IN ASSOCIATION WITH PAIN INDUCTION}

Nuclear HMGB1 immunoreactivity is detected in most cells, including neurons, satellite cells, Schwann cells, microglia and astrocytes from naive mice and rats $(37,56,65,75)$ (Table 2$)$. Increased levels of HMGB1 mRNA and protein have been measured in several pain studies and have been interpreted to indicate HMGB1 release and involvement in nociception. However, it is important to note that pain-like behavior has also been reported in the absence of changes in HMGB1 protein levels. For example, total HMGB1 protein expression did not change in DRG after the application of autologous nucleus pulposus onto nerve roots (76), in the DRG after intraplantar injection of LPS to the paw (59) or in the spinal cord after the induction of CAIA (65) (Table 2). Because HMGB1 is abundant in the nucleus of most cells, a small increase in HMGB1 protein expression may be difficult to detect when analyzing whole cell-protein extracts. Focusing on the extracellular activities of HMGB1, the extent of HMGB1 translocation from the nucleus to the cytoplasm and extracellular space is a more relevant indicator of release. Accordingly, in several studies, subcellular fractionation examining the presence of HMGB1 in the extranuclear fraction and immunohistochemistry have been used to determine whether there is increased translocation out of the nucleus. After tibial nerve injury and spinal nerve ligation, immunohistochemistry showed that the increased levels of HMGB1 found in DRG wholecell homogenates were associated with translocation from the nucleus to the cytoplasm $(37,56)$. Notably, in the arthritis model, where no change in HMGB1 pro- 
tein levels were found in spinal cord whole-cell homogenate, the HMGB1 levels were found to be elevated during ongoing joint inflammation when the extranuclear fraction was analyzed (65) (Table 2). Thus, the later study indicates that increased translocation of HMGB1 from the nucleus to the cytoplasm can take place even in the absence of signs of increased production of HMGB1 protein based on analysis of whole-cell homogenates. Another important aspect of HMGB1 analysis is that most methods used for detection are antibody-based (Western blotting, enzyme-linked immunosorbent assay, enzyme-linked immunospot (ELISPOT) and immunohistochemistry), and these do not distinguish between the different redox forms of HMGB1, or whether HMGB1 has been subjected to other posttranslational modifications, or if HMGB1 is in complex with other factors. Future studies using alternative methodology that enables such characterization, for example, liquid chromatography-tandem mass spectrometry analysis (92), promises to advance our understanding of the nature of HMGB1 involvement and receptor dependence in pathological pain.

\section{HMGB 1 AS A POTENTIAL THERAPEUTIC TARGET FOR CHRONIC PAIN}

Accumulating data support the role of HMGB1 as an important inflammatory pronociceptive mediator, which can act alone or in complex with several other molecules. Much knowledge has already been accrued by in vitro and in vivo studies, deciphering the pathways, receptors and posttranslational modifications that determine the consequences of HMGB1 release. As shown in Table 1, these insights have been used in preclinical studies in which different agents that prevent the actions of HMGB1 have proven to reduce hypersensitivity in animal models of pathology-induced pain. Of course, to use these agents as a pain-relieving drug in humans, more studies need to be performed. It is noteworthy, however, that some drugs that are already in clinical use have been attributed to a HMGB1 blocking effect. As mentioned earlier in this review, glycyrrhizin and thrombomodulin are used as antiviral and anticoagulant agents, respectively, and also attenuate pain-like behavior in preclinical studies. In addition, gold salts were one of the first widely used therapies for rheumatoid arthritis and are shown to inhibit HMGB1 translocation from the nucleus to the cytoplasm in macrophages (93). Toxicity, however, has limited the use of gold salts, but it has been proposed that gold nanoparticles use the same mechanism as gold salts (94). Oxaliplatin is another compound that retains HMGB1 in the nucleus and demonstrates beneficial effects in experimental arthritis (95). This agent is in use as an anticancer drug, but unfortunately also induces signs of neuropathic pain in animals $(96,97)$. Moreover, metformin, a known antidiabetic drug, reduces LPS-induced HMGB1 release from macrophages and hyperglycemia-induced HMGB1 production in cardiomyocytes. Of note, metformin also reduces nerve injury-induced hypersensitivity (98).

Although it is promising that the blockade of HMGB1 effects is part of the pharmacological profile of the drugs mentioned above, they are also assigned other mechanisms of action that could be the dominating reason for their clinical effect and also contribute to their side effects. A more direct approach would be to block the actions of HMGB1 by using neutralizing antibodies or other agents that specifically interfere with HMGB1, or perhaps preferentially, specific HMGB1 isoforms. The studies described in this review provide proof of concept that antibody-directed HMGB1 blockade reduces pathological pain in preclinical settings. Hopefully, it will be possible in the future to translate the progress made in animal studies into pain-relieving therapeutics for clinical trials in humans. Although not as specific, another approach would be to use antagonists against the different HMGB1 receptors. For this, as well as for a general understanding of the role of HMGB1 in pain, it will be important to delineate which re- ceptors and which cells are stimulated by HMGB1 released in conjunction with the induction of a painful condition. Moreover, answering questions that address the specific contributions of the respective HMGB1 receptors and HMGB1 redox forms in different states of persistent pain, and whether there are changes over time, will provide important clues into how to target HMGB1 as a treatment for debilitating and refractory pain.

\section{CONCLUSIONS}

HMGB1 is a key factor in the pathogenesis of a wide variety of inflammatory conditions and has also been identified as a mediator of neuroinflammation. Experimental studies suggest that HMGB1 is released both peripherally and spinally in models of pathological pain and that it can drive nociceptive signaling via actions on sensory neuronal and/or glia and immune cells. Of importance, systemic and intrathecal administration of HMGB1 blocking agents reverse pain-like behavior, indicating that HMGB1 has pronociceptive properties both in the peripheral and central nervous system. Emerging evidence indicates that TLR- and RAGE-induced signaling contribute to pain hypersensitivity. Because HMGB1 is an endogenous multireceptor ligand for these receptors and plays a pivotal role in chronic pain, it serves as a critical link between sterile inflammation, pattern-recognition receptors and activation of the sensory nervous system. As such, HMGB1 presents a new target of therapy for pain in inflammatory and neuropathic pain conditions. Future studies are warranted to define the pathways for HMGB1 release and actions in pain conditions and to elucidate the most effective strategies for blocking the effect of this mediator.

\section{DISCLOSURE}

The authors declare that they have no competing interests as defined by Molecular Medicine, or other interests that might be perceived to influence the results and discussion reported in this paper. 


\section{REFERENCES}

1. Ueda T, Yoshida M. (2010) HMGB proteins and transcriptional regulation. Biochim. Biophys. Acta. 1799:114-8.

2. Andersson U, Tracey KJ. (2011) HMGB1 is a therapeutic target for sterile inflammation and infection. Annu. Rev. Immunol. 29:139-62.

3. Harris HE, Andersson U, Pisetsky DS. (2012) HMGB1: a multifunctional alarmin driving autoimmune and inflammatory disease. Nat. Rev. Rheumatol. 8:195-202.

4. Park JS, et al. (2004) Involvement of toll-like receptors 2 and 4 in cellular activation by high mobility group box 1 protein. J. Biol. Chem. 279:7370-7.

5. van Beijnum JR, Buurman WA, Griffioen AW. (2008) Convergence and amplification of toll-like receptor (TLR) and receptor for advanced glycation end products (RAGE) signaling pathways via high mobility group B1 (HMGB1). Angiogenesis. 11:91-9.

6. Wang H, et al. (1999) HMG-1 as a late mediator of endotoxin lethality in mice. Science. 285:248-51.

7. Maeda T, Ozaki M, Kobayashi Y, Kiguchi N, Kishioka S. (2013) HMGB1 as a potential therapeutic target for neuropathic pain. J. Pharmacol. Sci. 123:301-5.

8. Woolf CJ, Salter MW. (2000) Neuronal plasticity: increasing the gain in pain. Science. 288:1765-9.

9. Zeilhofer HU. (2005) Synaptic modulation in pain pathways. Rev. Physiol. Biochem. Pharmacol. 154:73-100.

10. Xu Q, Yaksh TL. (2011) A brief comparison of the pathophysiology of inflammatory versus neuropathic pain. Curr. Opin. Anaesthesiol. 24:400-7.

11. Costigan M, Scholz J, Woolf CJ. (2009) Neuropathic pain: a maladaptive response of the nervous system to damage. Annu. Rev. Neurosci. 32:1-32.

12. Gangadharan V, Kuner R. (2013) Pain hypersensitivity mechanisms at a glance. Dis. Model Mech. 6:889-95.

13. McMahon SB, Malcangio M. (2009) Current challenges in glia-pain biology. Neuron. 64:46-54.

14. Basbaum AI, Bautista DM, Scherrer G, Julius D. (2009) Cellular and molecular mechanisms of pain. Cell. 139:267-84

15. Woolf CJ. (2010) What is this thing called pain? J. Clin. Invest. 120:3742-4.

16. Latremoliere A, Woolf CJ. (2009) Central sensitization: a generator of pain hypersensitivity by central neural plasticity. J. Pain. 10:895-926.

17. Dublin P, Hanani M. (2007) Satellite glial cells in sensory ganglia: their possible contribution to inflammatory pain. Brain Behav. Immun. 21:592-8.

18. Zhang H, et al. (2009) Altered functional properties of satellite glial cells in compressed spinal ganglia. Glia. 57:1588-99.

19. Takeda M, et al. (2007) Enhanced excitability of nociceptive trigeminal ganglion neurons by satellite glial cytokine following peripheral inflammation. Pain. 129:155-66.

20. Svensson CI, Brodin E. (2010) Spinal astrocytes in pain processing: non-neuronal cells as therapeutic targets. Mol. Interv. 10:25-38.
21. Ji RR, Berta T, Nedergaard M. (2013) Glia and pain is chronic pain a gliopathy? Pain. 154 Suppl 1: S10-28.

22. Grace PM, Hutchinson MR, Maier SF, Watkins LR. (2014) Pathological pain and the neuroimmune interface. Nat. Rev. Immunol. 14:217-31.

23. Tsung A, et al. (2007) HMGB1 release induced by liver ischemia involves Toll-like receptor 4 dependent reactive oxygen species production and calcium-mediated signaling. J. Exp. Med. 204:2913-23.

24. Scaffidi P, Misteli T, Bianchi ME. (2002) Release of chromatin protein HMGB1 by necrotic cells triggers inflammation. Nature. 418:191-5.

25. Gardella S, et al. (2002) The nuclear protein HMGB1 is secreted by monocytes via a nonclassical, vesicle-mediated secretory pathway. EMBO Rep. 3:995-1001.

26. Kang R, et al. (2014) HMGB1 in health and disease. Mol. Aspects Med. 40C:1-116.

27. Jiang W, Pisetsky DS. (2006) The role of IFNalpha and nitric oxide in the release of HMGB1 by RAW 264.7 cells stimulated with polyinosinicpolycytidylic acid or lipopolysaccharide. J. Immunol. 177:3337-43.

28. Lu B, et al. (2014) JAK/STAT1 signaling promotes HMGB1 hyperacetylation and nuclear translocation. Proc. Natl. Acad. Sci. U. S. A. 111:3068-73.

29. Rendon-Mitchell B, et al. (2003) IFN-gamma induces high mobility group box 1 protein release partly through a TNF-dependent mechanism. J. Immunol. 170:3890-7.

30. Zhou JR, et al. (2013) Neuropeptide Y induces secretion of high-mobility group box 1 protein in mouse macrophage via PKC/ERK dependent pathway. J. Neuroimmunol. 260:55-9.

31. Bonaldi T, et al. (2003) Monocytic cells hyperacetylate chromatin protein HMGB1 to redirect it towards secretion. EMBO J. 22:5551-60.

32. Ito I, Fukazawa J, Yoshida M. (2007) Post-translational methylation of high mobility group box 1 (HMGB1) causes its cytoplasmic localization in neutrophils. J. Biol. Chem. 282:16336-44.

33. Zhang X, et al. (2008) Calcium/calmodulindependent protein kinase (CaMK) IV mediates nucleocytoplasmic shuttling and release of HMGB1 during lipopolysaccharide stimulation of macrophages. J. Immunol. 181:5015-23.

34. Ma L, Kim SJ, Oh KI. (2012) Calcium/calmodulindependent protein kinase is involved in the release of high mobility group box 1 via the interferonbeta signaling pathway. Immune Netw. 12:148-54.

35. Oh YJ, et al. (2009) HMGB1 is phosphorylated by classical protein kinase $C$ and is secreted by a calcium-dependent mechanism. J. Immunol. 182:5800-9

36. Lu B, et al. (2012) Novel role of PKR in inflammasome activation and HMGB1 release. Nature. 488:670-4.

37. Feldman P, Due MR, Ripsch MS, Khanna R, White FA. (2012) The persistent release of HMGB1 contributes to tactile hyperalgesia in a rodent model of neuropathic pain. J. Neuroinflammation. 9:180
38. Allette YM, et al. (2014) Identification of a functional interaction of HMGB1 with receptor for advanced glycation end-products in a model of neuropathic pain. Brain Behav. Immun. 42:169-77.

39. Pedrazzi M, et al. (2012) Potentiation of NMDA receptor-dependent cell responses by extracellular high mobility group box 1 protein. PLoS One. 7:e44518.

40. Balosso S, Liu J, Bianchi ME, Vezzani A. (2014) Disulfide-containing high mobility group box-1 promotes N-methyl-D-aspartate receptor function and excitotoxicity by activating Toll-like receptor 4-dependent signaling in hippocampal neurons. Antioxid. Redox. Signal. 21:1726-40.

41. Chiavegato A, Zurolo E, Losi G, Aronica E, Carmignoto G. (2014) The inflammatory molecules IL-1 $\beta$ and HMGB1 can rapidly enhance focal seizure generation in a brain slice model of temporal lobe epilepsy. Front. Cell. Neurosci. 8:155

42. Hori O, et al. (1995) The receptor for advanced glycation end products (RAGE) is a cellular binding site for amphoterin: mediation of neurite outgrowth and co-expression of rage and amphoterin in the developing nervous system. J. Biol. Chem. 270:25752-61.

43. Ivanov S, et al. (2007) A novel role for HMGB1 in TLR9-mediated inflammatory responses to CpGDNA. Blood. 110:1970-81.

44. Chen GY, Tang J, Zheng P, Liu Y. (2009) CD24 and Siglec-10 selectively repress tissue damageinduced immune responses. Science. 323:1722-5.

45. Orlova VV, et al. (2007) A novel pathway of HMGB1-mediated inflammatory cell recruitment that requires Mac-1-integrin. EMBO J. 26:1129-39.

46. Sha Y, Zmijewski J, Xu Z, Abraham E. (2008) HMGB1 develops enhanced proinflammatory activity by binding to cytokines. J. Immunol. 180:2531-7.

47. Campana L, Bosurgi L, Bianchi ME, Manfredi AA, Rovere-Querini P. (2009) Requirement of HMGB1 for stromal cell-derived factor1/CXCL12-dependent migration of macrophages and dendritic cells. J. Leukoc. Biol. 86:609-15.

48. Hreggvidsdottir HS, et al. (2012) High mobility group box protein 1 (HMGB1)-partner molecule complexes enhance cytokine production by signaling through the partner molecule receptor. Mol. Med. 18:224-30.

49. Venereau E, et al. (2012) Mutually exclusive redox forms of HMGB1 promote cell recruitment or proinflammatory cytokine release. J. Exp. Med. 209:1519-28.

50. Yang H, et al. (2012) Redox modification of cysteine residues regulates the cytokine activity of high mobility group box-1 (HMGB1). Mol. Med. 18:250-9.

51. Huttunen HJ, Fages C, Kuja-Panula J, Ridley AJ, Rauvala H. (2002) Receptor for advanced glycation end products-binding $\mathrm{COOH}$-terminal motif of amphoterin inhibits invasive migration and metastasis. Cancer Res. 62:4805-11.

52. McMahon SB, Cafferty WB, Marchand F. (2005) Immune and glial cell factors as pain mediators and modulators. Exp. Neurol. 192:444-62. 
53. Wadachi R, Hargreaves KM. (2006) Trigeminal nociceptors express TLR-4 and CD14: a mechanism for pain due to infection. J. Dent. Res. 85:49-53.

54. Li Y, et al. (2014) Toll-like receptor 4 signaling contributes to Paclitaxel-induced peripheral neuropathy. J. Pain. 15:712-25.

55. Due MR, et al. (2012) Neuroexcitatory effects of morphine-3-glucuronide are dependent on Toll-like receptor 4 signaling. J. Neuroinflammation. 9:200.

56. Shibasaki M, et al. (2010) Induction of high mobility group box-1 in dorsal root ganglion contributes to pain hypersensitivity after peripheral nerve injury. Pain. 149:514-21.

57. Nicotra L, Loram LC, Watkins LR, Hutchinson MR. (2012) Toll-like receptors in chronic pain. Exp. Neurol. 234:316-29.

58. Chacur M, et al. (2001) A new model of sciatic inflammatory neuritis (SIN): induction of unilateral and bilateral mechanical allodynia following acute unilateral peri-sciatic immune activation in rats. Pain. 94:231-44.

59. Tanaka J, et al. (2013) Recombinant human soluble thrombomodulin prevents peripheral HMGB1-dependent hyperalgesia in rats. Br. J. Pharmacol. 170:1233-41.

60. Milligan ED, et al. (2003) Spinal glia and proinflammatory cytokines mediate mirror-image neuropathic pain in rats. J. Neurosci. 23:1026-40.

61. Tanaka J, et al. (2014) Bladder pain relief by HMGB1 neutralization and soluble thrombomodulin in mice with cyclophosphamideinduced cystitis. Neuropharmacology. 79:112-8.

62. Kokkola R, et al. (2002) High mobility group box chromosomal protein 1: a novel proinflammatory mediator in synovitis. Arthritis Rheum. 46:2598-603.

63. Pullerits R, et al. (2003) High mobility group box chromosomal protein 1, a DNA binding cytokine, induces arthritis. Arthritis Rheum. 48:1693-700.

64. O'Connor KA, et al. (2003) Further characterization of high mobility group box 1 (HMGB1) as a proinflammatory cytokine: central nervous system effects. Cytokine. 24:254-65.

65. Agalave NM, et al. (2014) Spinal HMGB1 induces TLR4-mediated long-lasting hypersensitivity and glial activation and regulates pain-like behavior in experimental arthritis. Pain. 155:1802-13.

66. Hoppe G, Talcott KE, Bhattacharya SK, Crabb JW, Sears JE. (2006) Molecular basis for the redox control of nuclear transport of the structural chromatin protein Hmgb1. Exp. Cell. Res. 312:3526-38.

67. Calogero S, et al. (1999) The lack of chromosomal protein Hmg1 does not disrupt cell growth but causes lethal hypoglycaemia in newborn mice. Nat. Genet. 22:276-80.

68. Kang R, et al. (2014) Intracellular Hmgb1 inhibits inflammatory nucleosome release and limits acute pancreatitis in mice. Gastroenterology. 146:1097-107.

69. Yanai H, et al. (2013) Conditional ablation of HMGB1 in mice reveals its protective function against endotoxemia and bacterial infection. Proc. Natl. Acad. Sci. U. S. A. 110:20699-704

70. Kokkola R, et al. (2003) Successful treatment of collagen-induced arthritis in mice and rats by targeting extracellular high mobility group box chromosomal protein 1 activity. Arthritis Rheum. 48:2052-8.

71. Andersson U, Erlandsson-Harris H, Yang H, Tracey KJ. (2002) HMGB1 as a DNA-binding cytokine. J. Leukoc. Biol. 72:1084-91.

72. Yang H, et al. (2004) Reversing established sepsis with antagonists of endogenous high-mobility group box 1. Proc. Natl. Acad. Sci. U. S. A. 101:296-301.

73. Mollica L, et al. (2007) Glycyrrhizin binds to high-mobility group box 1 protein and inhibits its cytokine activities. Chem. Biol. 14:431-41.

74. Abeyama K, et al. (2005) The N-terminal domain of thrombomodulin sequesters high-mobility group-B1 protein, a novel antiinflammatory mechanism. J. Clin. Invest. 115:1267-74.

75. Nakamura Y, et al. (2013) Neuropathic pain in rats with a partial sciatic nerve ligation is alleviated by intravenous injection of monoclonal antibody to high mobility group box-1. PLoS One. 8:e73640.

76. Otoshi K, Kikuchi S, Kato K, Sekiguchi M, Konno S. (2011) Anti-HMGB1 neutralization antibody improves pain-related behavior induced by application of autologous nucleus pulposus onto nerve roots in rats. Spine (Phila $\mathrm{Pa}$ 1976). 36:E692-8.

77. Wahamaa $\mathrm{H}$, et al. (2011) High mobility group box protein 1 in complex with lipopolysaccharide or IL-1 promotes an increased inflammatory phenotype in synovial fibroblasts. Arthritis Res. Ther. 13:R136.

78. Hreggvidsdottir HS, et al. (2009) The alarmin HMGB1 acts in synergy with endogenous and exogenous danger signals to promote inflammation. J. Leukoc. Biol. 86:655-62.

79. Tong W, et al. (2010) Spinal high-mobility group box 1 contributes to mechanical allodynia in a rat model of bone cancer pain. Biochem. Biophys. Res. Commun. 395:572-6.

80. Ren PC, et al. (2012) High-mobility group box 1 contributes to mechanical allodynia and spinal astrocytic activation in a mouse model of type 2 diabetes. Brain Res. Bull. 88:332-7.

81. Bas DB, et al. (2012) Collagen antibody-induced arthritis evokes persistent pain with spinal glial involvement and transient prostaglandin dependency. Arthritis Rheum. 64:3886-96.

82. Su J, et al. (2015) Phenotypic changes in dorsal root ganglion and spinal cord in the collagen antibody-induced arthritis mouse model. J. Comp. Neurol. 2015, Jan 29 [Epub ahead of print].

83. Kim ID, Lee JK. (2013) HMGB1-binding heptamer confers anti-inflammatory effects in primary microglia culture. Exp. Neurobiol. 22:301-7.

84. Kim SW, et al. (2012) Glycyrrhizic acid affords robust neuroprotection in the postischemic brain via anti-inflammatory effect by inhibiting HMGB1 phosphorylation and secretion. Neurobiol. Dis. 46:147-56.

85. Shin JH, Lee HK, Lee HB, Jin Y, Lee JK. (2014) Ethyl pyruvate inhibits HMGB1 phosphorylation and secretion in activated microglia and in the postischemic brain. Neurosci. Lett. 558:159-63.

86. Zurolo E, et al. (2011) Activation of Toll-like receptor, RAGE and HMGB1 signalling in malformations of cortical development. Brain. 134:1015-32

87. Kim JB, et al. (2006) HMGB1, a novel cytokinelike mediator linking acute neuronal death and delayed neuroinflammation in the postischemic brain. J. Neurosci. 26:6413-21.

88. Laird MD, et al. (2014) High mobility group box protein-1 promotes cerebral edema after traumatic brain injury via activation of toll-like receptor 4. Glia. 62:26-38.

89. Pedrazzi M, et al. (2007) Selective proinflammatory activation of astrocytes by high-mobility group box 1 protein signaling. J. Immunol. 179:8525-32.

90. Qiu J, et al. (2010) High-mobility group box 1 promotes metalloproteinase- 9 upregulation through Toll-like receptor 4 after cerebral ischemia. Stroke. 41:2077-82.

91. Bonanno G, et al. (2007) The high-mobility group box 1 cytokine induces transporter-mediated release of glutamate from glial subcellular particles (gliosomes) prepared from in situ-matured astrocytes. Int. Rev. Neurobiol. 82:73-93.

92. Antoine DJ, et al. (2009) High-mobility group box-1 protein and keratin-18, circulating serum proteins informative of acetaminophen-induced necrosis and apoptosis in vivo. Toxicol. Sci. 112:521-31.

93. Zetterstrom CK, et al. (2008) Pivotal advance: inhibition of HMGB1 nuclear translocation as a mechanism for the anti-rheumatic effects of gold sodium thiomalate. J. Leukoc. Biol. 83:31-8.

94. Tsai CY, et al. (2012) Size-dependent attenuation of TLR9 signaling by gold nanoparticles in macrophages. J. Immunol. 188:68-76.

95. Ostberg T, et al. (2008) Oxaliplatin retains HMGB1 intranuclearly and ameliorates collagen type IIinduced arthritis. Arthritis Res. Ther. 10:R1.

96. Ling B, Authier N, Balayssac D, Eschalier A, Coudore F. (2007) Behavioral and pharmacological description of oxaliplatin-induced painful neuropathy in rat. Pain. 128:225-34.

97. Ling B, et al. (2007) Behavioral and immunohistological assessment of painful neuropathy induced by a single oxaliplatin injection in the rat. Toxicology. 234:176-84.

98. Melemedjian OK, et al. (2011) Targeting adenosine monophosphate-activated protein kinase (AMPK) in preclinical models reveals a potential mechanism for the treatment of neuropathic pain. Mol. Pain. 7:70.

99. He Z, Guo Q, Xiao M, He C, Zou W. (2013) Intrathecal lentivirus-mediated transfer of interleukin-10 attenuates chronic constriction injuryinduced neuropathic pain through modulation of spinal high-mobility group box 1 in rats. Pain Physician. 16:E615-25.

100. Kuang X, et al. (2012) Effects of intrathecal epigallocatechin gallate, an inhibitor of Toll-like receptor 4, on chronic neuropathic pain in rats. Eur. J. Pharmacol. 676:51-6. 